\title{
RELIABILITY OF ASSESSING BALLET DANCERS' POSTURAL STABILITY IN THE UNSHOD AND THE EN POINTE RELEVÉ POSITION WITH A SMARTPHONE APPLICATION
}

original paper

() Wroclaw University of Health and Sport Sciences

DOI: https://doi.org/10.5114/hm.2022.109069

\section{FANI PADERI, ANALINA EMMANOUIL, ELISSAVET ROUSANOGLOU}

Sport Biomechanics Lab, Sector of Sports Medicine and Biology of Exercise, School of Physical Education and Sport Science, National and Kapodistrian University of Athens, Athens, Greece

\begin{abstract}
Purpose. This study aimed to verify the reliability of estimating ballet dancers' postural stability during the unshod and the en pointe relevé position with a smartphone application

Methods. The participants (13 ballet dancers, $22.4 \pm 2.5$ years of age) were tested in the unshod and the en pointe relevé position (YMED Balance Test application, smartphone secured at the L5 level for centre of mass approximation, 10 trials for each condition, 10 seconds per trial, 2-minute intertrial break, arms relaxed at bodyside, gaze fixated at an eye-level target, preferred feet width and orientation). Paired t-tests examined the inter-condition differences. Relative (intraclass correlation coefficient, ICC) and absolute (standard error of measurement, SEM, SEM\%) reliability indices (for accumulated and paired trials) were computed for each condition (SPSS software v. 26.0, $p<0.05$ ).

Results. The total balance score and all centre of mass spatial measures indicated worse postural stability in the en pointe condition $(p<0.05)$, with no significant temporal differences $(p>0.05)$. The total body balance score was the most reliable measure (good to excellent ICCs, low to moderate SEM\%) with a minimum of 8 trials ensuring reliability in both the unshod and the en pointe relevé positions.

Conclusions. Taken a minimum of 8 trials and the measure of total balance score, we may obtain a reliable estimation of ballet dancers' postural stability in the unshod and the en pointe relevé position by using the YMED Balance Test smartphone application.
\end{abstract}

Key words: balance, centre of mass, tip-toe stance, accelerometer, inertial sensors

\section{Introduction}

The postural stability of ballet dancers has been evaluated in a variety of stances [1-5]. The en pointe relevé position is an ultimate postural stability challenge for ballet dancers owing to the extreme ankle joint configuration (full plantar flexion) [6], which together with the very small base of support provided by the pointe shoes induces a very sensitive equilibrium and an increased falling risk [1]. Nevertheless, the en pointe relevé position is not common among the relevant postural stability studies in ballet dancers. Evaluation of ballet dancers' postural stability in the en pointe relevé position allows to determine the skill level and may contribute to specified training technical recommendations [2].
The gold standard of postural stability evaluation in a laboratory setting is the force plate in general population studies [7] but also among ballet dancers [2, 5], followed by elaborated motion capture systems $[6,8,9]$. In recent years, wearable sensors based on miniaturized inertial measurement units (typically including accelerometers and gyroscopes) have increasingly been used in postural stability studies [7, 10-12], ballet dancers studies inclusive [3, 13].

Although the laboratory devices provide a state-ofthe-art quantitative evaluation of postural stability, they involve a high cost in both money and time, the evaluation procedure is complex, and a field setting is often not applicable because of the limited portability of the devices [14-16]. Smartphones can be regarded as a solution for overcoming the limitations of laboratory-based

Correspondence address: Elissavet Rousanoglou, School of Physical Education and Sport Science, National and Kapodistrian University of Athens, Ethnikis Antistasis 41, Daphne, 172-37, Athens, Greece, e-mail: erousan@phed.uoa.gr

Received: March 30, 2021

Accepted for publication: August 8, 2021

Citation: Paderi F, Emmanouil A, Rousanoglou E. Reliability of assessing ballet dancers' postural stability in the unshod and the en pointe relevé position with a smartphone application. Hum Mov. 2022;23(2):84-96; doi: https://doi.org/10.5114/ hm.2022.109069. 
assessments. Portability, together with their built-in accelerometers, have turned smartphones into a major potential for objective and convenient (easy, portable, and affordable) postural stability assessment in a field setting [14, 16-18]. In such a case, the reliability of the smartphone application itself, as well as of the particular standing position and the examinee specificity remains an issue of concern $[9,15,17]$. Thus, the purpose of this study was to evaluate the reliability of determining ballet dancers' postural stability during the unshod and the en pointe relevé position by using a smartphone application.

\section{Material and methods}

\section{Sample}

Thirteen young women (age: $22.4 \pm 2.5$ years, body height: $162.6 \pm 5.95 \mathrm{~cm}$, body mass: $54.62 \pm 8.03 \mathrm{~kg}$ ) experienced in classic ballet dance (11.8 \pm 4.1 years), as well as in the en pointe relevé position $(5.6 \pm 3.1$ years) participated in the study. They all used their regular pointe shoes as the time of use does not appear to affect stability [19]. The participants were free of musculoskeletal injuries or vestibular disorders that would potentially affect their postural stability.

\section{Smartphone application}

The YMED Balance Test application for an Androidbased smartphone (Xiaomi Redmi Note 5, released in February 2018, https://www.mi.com/global/redminote-5/specs/) was used in the present study. The Xiaomi Redmi Note 5 smartphone has an accelerometer sensor resolution at $0.0024 \mathrm{~m} / \mathrm{s}^{2}$, which is a common one among the smartphone accelerometer sensors (https://phyphox.org/sensordb/). The application (developed in 2012 by Physiotools YMED, http://physiotool.blogspot.com/2011/) uses only the accelerometer sensor embedded in smartphones and is available from the Google Play Store (https://play.google.com/store/ apps/details?id=com.hyunc.rehatrain.balancecheck $\& \mathrm{hl}=\mathrm{el} \& \mathrm{gl}=\mathrm{US}$ ).

The YMED Balance Test was selected from among 3 smartphone applications (Balance Test, Sway, and YMED Balance Test) [17]. The selection criteria were the low cost together with the specificity of the offered tasks, which allows the assessment of static postural stability through an easy and non-expensive software acquisition. Specifically, among the 3 smartphone applications, the Balance Test by Slani is free of cost but designed as a balance board test, and the Sway has to be paid by a minimum of 50 profiles per year $(\$ 4.50$ per profile) [17]. Instead, the YMED Balance Test can be used completely through a final $\$ 8.00$ payment, without having to pay again in the future [17]. Furthermore, it is reported to provide values of moderate to high relative reliability with intraclass correlation coefficients (ICCs) ranging from 0.62 to 0.91 [17], and has been validated against the Biodex Stability System with a rather low systematic error at 0.01-0.08 [15].

\section{Data collection procedure}

The YMED Balance Test offers 4 types of tasks (vestibule, sitting, knee, balance board) with a 10-second data collection period. In their validation study against the Biodex Stability System, Park et al. [15] report its sampling frequency as 1 dot every $60 \mathrm{~ms}$ and 1000 dots per minute, which infers a sampling frequency of $16.6 \mathrm{~Hz}$. Since its first release in 2012 (personal communication with the developer atyhc0869@hanmail.net), the YMED Balance Test has been updated and the sampling frequency is $100 \mathrm{~Hz}$ (resulting in 1000 dots for the standardized 10-second sampling duration). The calibration of the accelerometer sensor of the smartphone was tested by using the RedPi Apps Tools (available from the Google Play Store at https:// play.google.com/store/apps/details?id=redpi.apps.ac celerometercalibrationfree\&hl=en\&gl=US). Also, the procedures described by Ma et al. [20] were applied for static bias testing.

The vestibule task of the YMED Balance Test was used in both the unshod and the en pointe relevé conditions. Data collection began via the start button, initiating the 5 -second zeroing calibration procedure (accompanied with a visual 5-4-3-2-1-START countdown), which is immediately followed by the 10 -second data collection. The first centre of mass $(\mathrm{CoM})$ point immediately after the completion of the 5 -second calibration procedure defines the zero CoM position (personal communication with the developer). After data collection, a report is saved on the mobile phone including the total balance score, as well as octadrant-based directional variables (Figure 1D). In both the unshod and the en pointe conditions, 3 familiarization trials were allowed per condition. Ten trials were performed in each condition (2-minute break among trials, 5-minute break before the main data collection procedure). In case the participants failed to maintain their relevé position throughout the 10-second data collection period, the trial was repeated. The subjects were instructed to stand in front of a ballet bar (typical height), place their hands on the bar, and remain in full contact 


\section{HUMAN MOVEMENT}

F. Paderi, A. Emmanouil, E. Rousanoglou, Smartphone reliability for ballet stability estimation
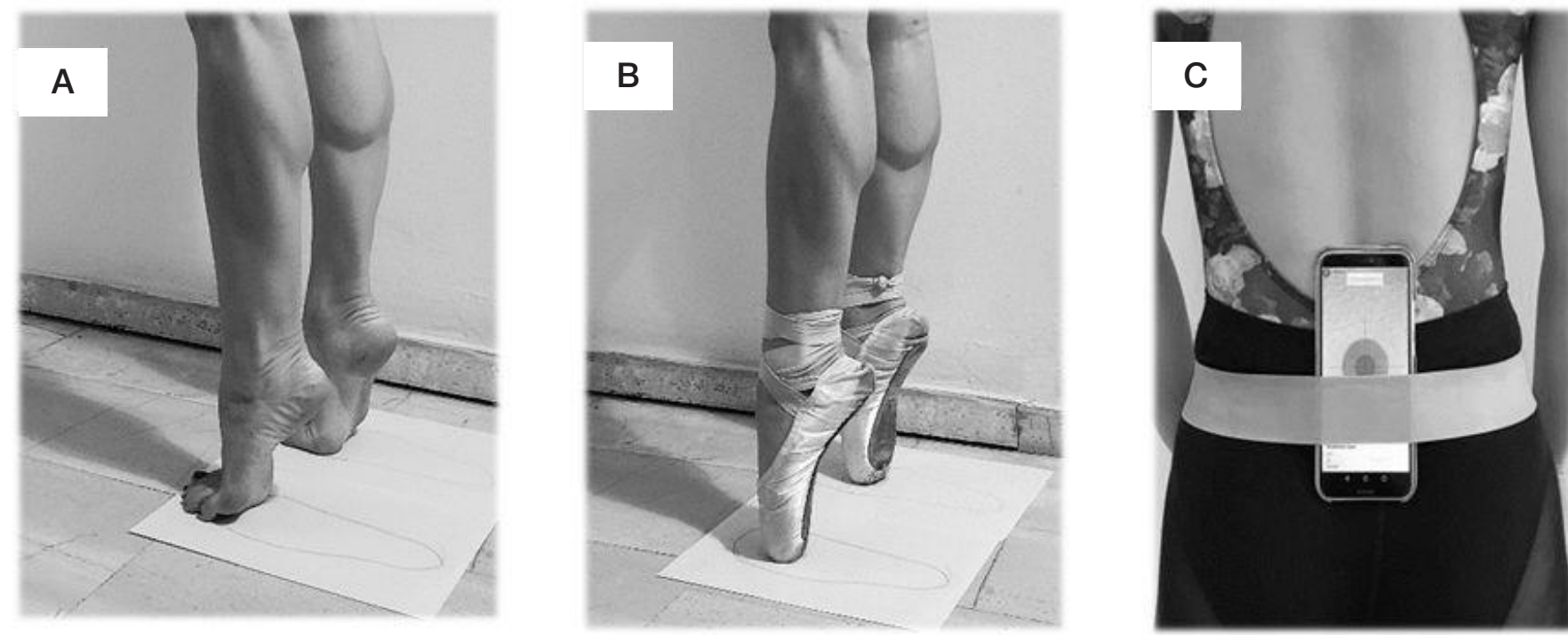

Total Balance Score YMED report

D 999.1

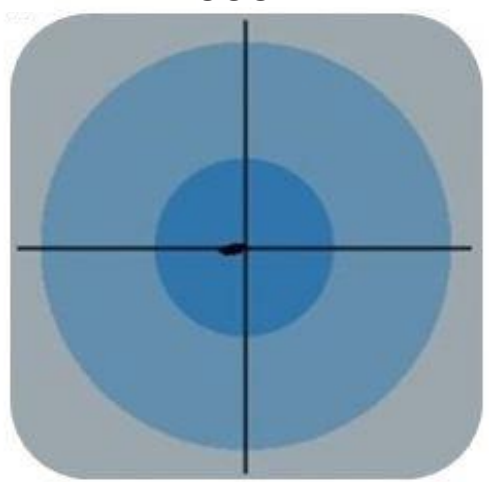

577.3

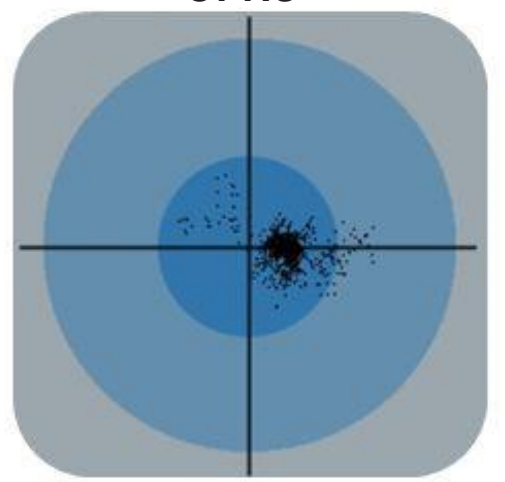

Directional Variables YMED report
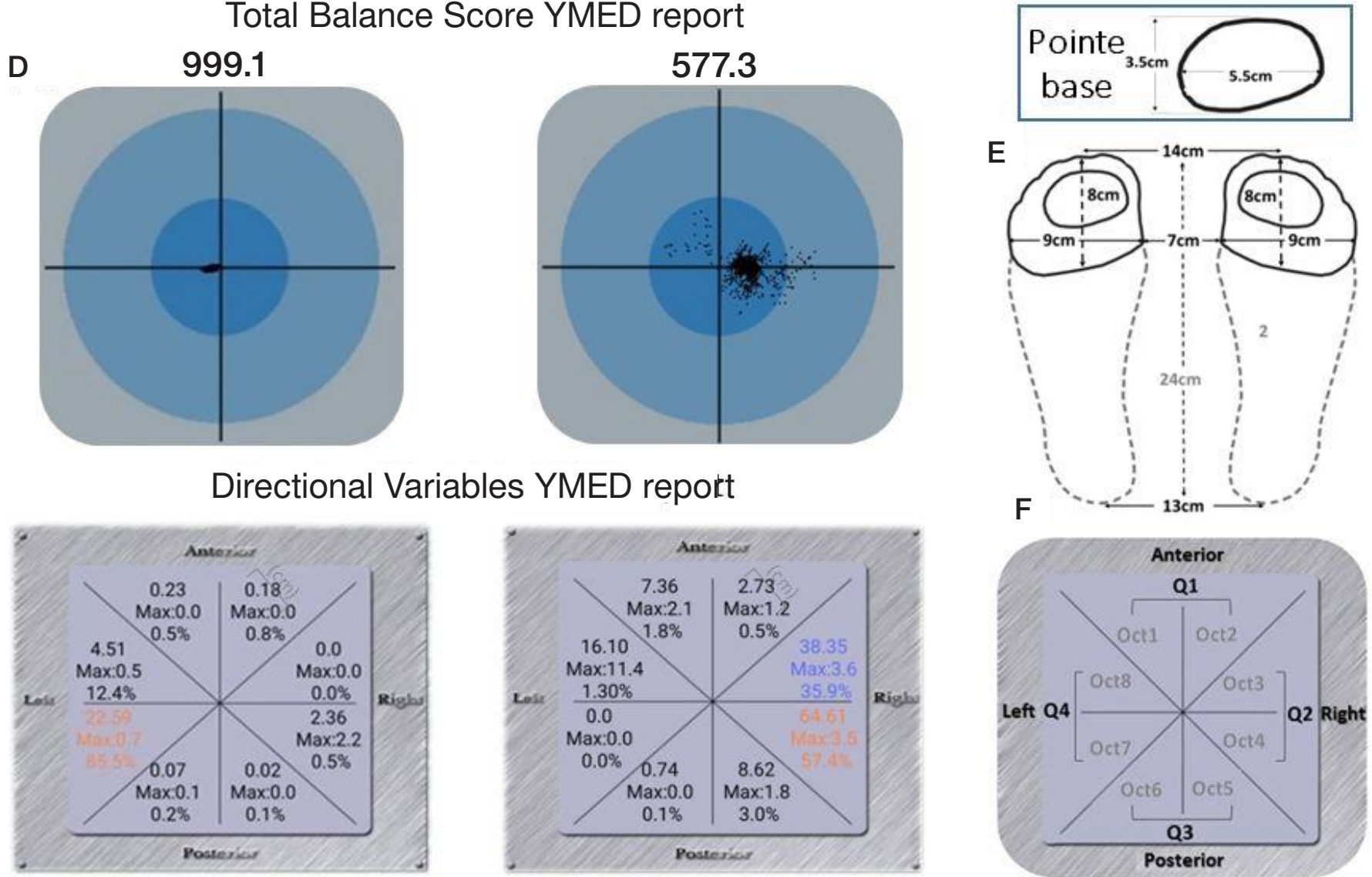

Figure. 1. Illustration of the unshod (A) and the en pointe (B) relevé stance, as well as the smartphone placement (C) To ensure the same feet repositioning in all trials, a piece of paper secured on the floor surface was used to outline the base of support. Panel D shows extracts of the YMED Balance Test reports saved in the smartphone for the total balance score (1 decimal point resolution), as well as for the directional variables of the sum $(\mathrm{cm})$, the maximum $(\mathrm{cm})$ ( 2 decimal points resolution), and the time percentage (\% ttotal, decimal point resolution, $100 \%=10$-second data collection duration). Red font indicates the octadrant where the peak directional values were recorded. Panel E illustrates the base of support outline for a representative participant (to facilitate visualization, the dotted lines indicate the full floor contact). Panel F presents the original directional octadrants (Oct) in which the YMED Balance Test provides the spatial and temporal directional variables of the centre of mass point displacement, and the directional quartiles (Q) used to facilitate the conceptualization of the centre of mass orientation results. 
Postural stability

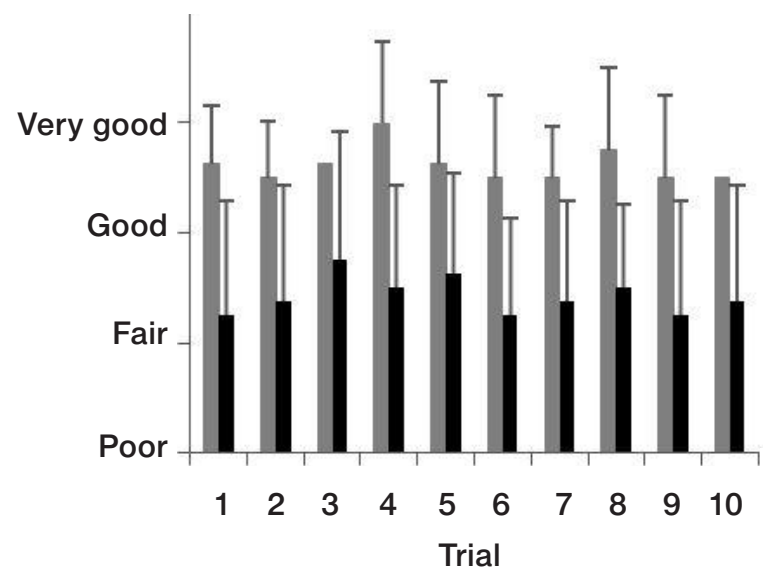

Fatigue

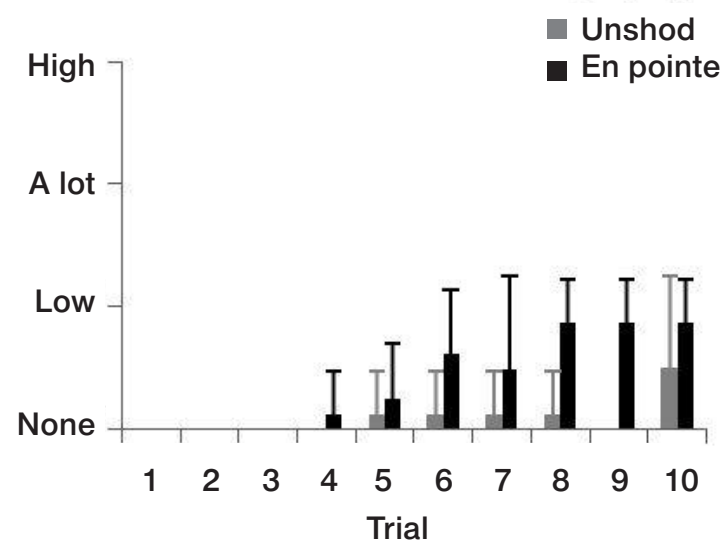

Figure 2. Participants' responses to the 4-point Likert scale concerning postural stability and perceived fatigue immediately after each trial of the unshod (grey bars) and the en pointe (black bars) relevé conditions

with the floor until instructed to adopt the relevé position. After adopting the relevé position (Figure 1A, B), a vocal cue informed them to slowly release the bar and relax their arms at the side of their body just before the calibration initiation (a vocal instruction was provided for gaze fixation on a target in front of them, at eye level). The mobile phone was securely positioned (narrow elastic ring band) on the dorsal body surface at the $\mathrm{O} 5$ level (i.e., approximating the CoM position) (Figure 1C). In both the unshod and the en pointe condition, the perimeter of the initial feet placement (preferred width and orientation) was outlined on a piece of paper secured on the floor, to ensure the same feet repositioning in all trials (Figure 1E).

After each one of the trials, all participants rated their postural stability and sense of fatigue using a 4-point Likert scale. The ratings (Figure 2) indicated that the postural stability in the en pointe relevé was about 50\% lower than in the unshod relevé. Despite an increasing trend after the $6^{\text {th }}$ trial in the en pointe relevé, the fatigue ratings did not exceed the low level. Overall, the Likert scale ratings allow the inference of no learning or fatigue effect along with the 10 trials in both the unshod and the en pointe relevé. The presence of a learning and/or fatigue effect was further examined through statistical procedures; these are described in the statistics section and their outcomes are presented in the results section.

\section{Variables}

The YMED Balance Test provides a total postural stability estimation (total balance score with 1 decimal point resolution) ranging from 0 to 1000 (0: worst bal- ance, 1000: best balance). The YMED Balance Test (personal communication with the developer) estimates the total balance score from the CoM point displacement within 10 concentric circular zones, with zero CoM position defining the centre of the concentric circular zones, and the inner and outer zone indicating the better and the worse stability, respectively. It also provides spatial and temporal data for the CoM point orientation within octadrant zones, that is, the sum of the CoM point displacements $(\mathrm{cm}, 2$ decimal points resolution), the maximum CoM point displacement (cm, 2 decimal points resolution), and the time percentage of the CoM point displacement ( $\%$ ttotal, $10 \mathrm{~s}=$ $100 \%, 1$ decimal point resolution), in each of the 8 octadrants. The 10-trial average consisted of the value of the balance score, as well as of the octadrant spatial and temporal values of the CoM point displacement. For conceptual facilitation of the CoM orientation, the octadrant values (Oct1, Oct2, Oct3, Oct4, Oct5, Oct6, Oct7, Oct8) were summed into quartile values indicating the anterior $(\mathrm{Q} 1=$ Oct1 + Oct2), rightwards $(\mathrm{Q} 2=$ Oct3 + Oct4), posterior $(\mathrm{Q} 3=$ Oct5 + Oct6), and leftwards $(\mathrm{Q} 4=$ Oct7 + Oct8) CoM point displacement (Figure 1F).

\section{Statistical analysis}

The statistical analysis included paired $t$-tests between the unshod and the en pointe relevé conditions, with the 10-trial average constituting the individual value (respectively for each of the examined variables) inserted in the statistical analysis. Data normality was not tested as with less than 30 cases such a test is not actually of usefulness, and a similar or even lower 
F. Paderi, A. Emmanouil, E. Rousanoglou, Smartphone reliability for ballet stability estimation

number of cases is not uncommon in other relevant studies ( $n=15$ [7], $n=10$ [21], $n=10$ [22], $n=5$ [23]). However, we applied the Spearman-Brown prophecy formula [24] to estimate the number of measurements (k) necessary to achieve the desired level of optimal reliability $\left(R_{\mathrm{k}}\right)$ :

$$
k=\left[R_{\mathrm{k}}^{*}(1-R)\right] /\left[R^{*}\left(1-R_{\mathrm{k}}\right)\right]
$$

The application of this formula in our study, with the desired level of reliability $\left(R_{\mathrm{k}}\right)$ set at $0.70,0.80$ and with $R$ being the value of the intraclass correlation coefficient (ICC) for the reliability between the $1^{\text {st }}$ and $2^{\text {nd }}$ trial ( $R=0.20$ and $R=0.80$ for the unshod and the en pointe, respectively), led to confirming the use of at least 9 trials in the unshod and just 2 trials in the en pointe relevé in this respect. As we aimed at a balanced design, we finally chose 10 trials for both relevé conditions.

Relative and absolute reliability measures were applied separately in the unshod and the en pointe relevé conditions. There are several forms of the ICC [25], but considering that the reliability that is in question concerns absolute agreement, the 2 -way random effects absolute agreement method, also known as $\operatorname{ICC}(2,1)$, was the most appropriate for the present study [21]. The ICC's upper and lower bounds of their 95\% confidence interval were also extracted. Relative reliability was classified in accordance with Fleiss [26] (ICC > 0.75: excellent, ICC between 0.40 and 0.75: fair to good, ICC < 0.40: poor). Absolute reliability was estimated by using the standard error of measurement (SEM) and the percentage SEM (SEM\%). SEM accounts for the within-subject variability, assesses how precisely a test measures a subject's true value, has the same units as the measure of interest, and is not sensitive to the between-subject variability of the data. Thus SEM, indicates the expected variation in observed values that occurs owing to the measurement error (if reliability $=0$, SEM will equal the standard deviation of the observed values; if test reliability $=1.00$, SEM will be zero). SEM was estimated as the square root of the mean square error term from ANOVA, as this estimation has the advantage of being independent of the specific ICC and allows more consistency in interpreting SEM values from different studies [27]. Furthermore, SEM\% allows a comparison of the expected variation in observed values between different conditions (as the 2 relevé conditions of the present study) and was defined as $(\mathrm{SEM} / \bar{x}) \times 100$, where $\bar{x}$ is the average of all observed values. Following the sug- gestion by Hopkins [28], the potential learning or fatigue effects on ICC and SEM were examined with separate analyses of accumulated trials ( $1^{\text {st }}$ to $2^{\text {nd }}, 1^{\text {st }}$ to $3^{\text {rd }}, \ldots, 1^{\text {st }}$ to $10^{\text {th }}$ ), as well as of consecutive pairs of trials $\left(1^{\text {st }}\right.$ vs. $2^{\text {nd }}, 2^{\text {nd }}$ vs. $3^{\text {rd }}, \ldots, 9^{\text {th }}$ vs. $\left.10^{\text {th }}\right)$. Also, in each relevé condition, we tested the trial effect (total of 10 trials) via repeated measures analysis, separately for each variable. If the Mauchly's test indicated a violation of data sphericity ( $p<0.05)$, the GreenhouseGeisser correction was used to estimate the trial effect significance. All statistical analyses were carried out with the IBM SPSS software v. 26.0, with the level of significance set at $\alpha=0.05$.

\section{Ethical approval}

The research related to human use has complied with all the relevant national regulations and institutional policies, has followed the tenets of the Declaration of Helsinki, and has been approved by the School of Physical Education and Sport Science, National and Kapodistrian University of Athens, Greece (approval protocol number: 1165/12-02-2020).

\section{Informed consent}

Informed consent has been obtained from all individuals included in this study.

\section{Results}

Comparison between unshod and en pointe relevé

Figure 3 presents the mean of accumulated trials, as well as its lower and upper bound of the $95 \%$ confidence intervals, for the total balance score and the spatial and temporal directional variables, for the total of 10 trials for testing the trial effect via repeated measures analysis, separately for each variable. No significant trial effect was found, either for the total balance score or for any of the spatial and temporal directional variables $(p>0.05)$.

The total balance score was $946.6 \pm 43.59$ in the unshod relevé, with a significantly lower value in the en pointe condition $(553.3 \pm 263.4)(t=5.053, d f=12$, $p<0.001$ ) (Figure 2). All spatial quartile measures were also significantly lower in the en pointe condition $(p<0.05)$, with no significant difference $(p>0.05)$ concerning the temporal measures (time percentage of the CoM displacement) (Figure 2). 


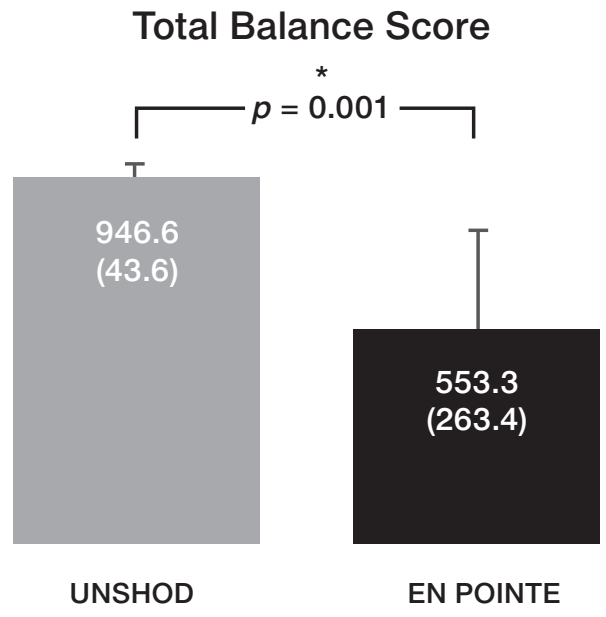

Sum of CoM point displacememt

Max of CoM point displacememt

\%Time of CoM point displacememt
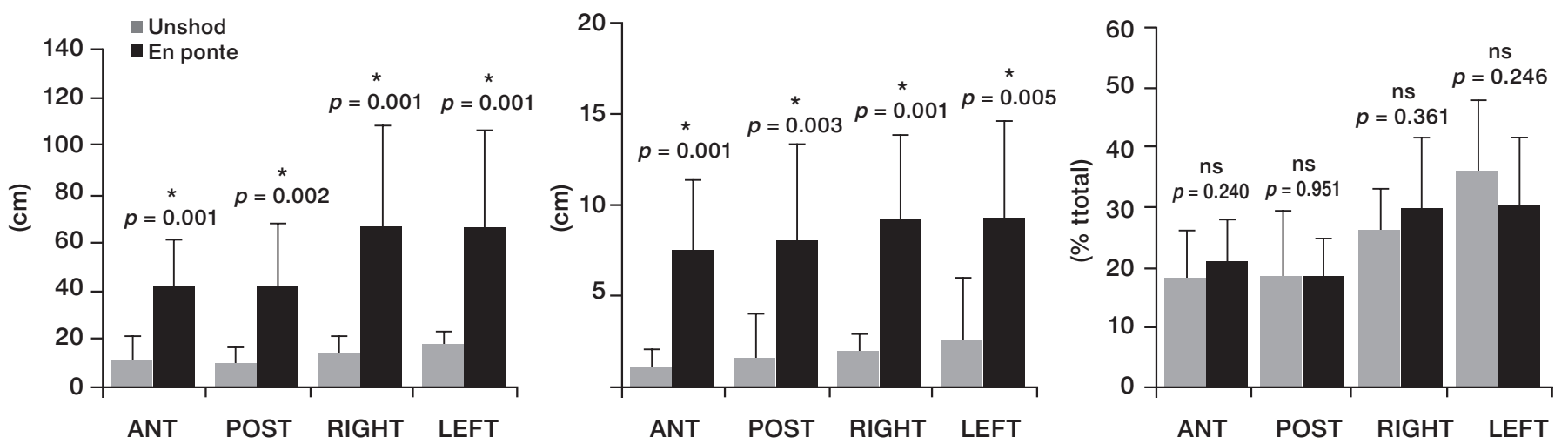

CoM - centre of mass, ttotal - total time, ns - non-significant difference at $p<0.05$

ANT - anteriorly, POST - posteriorly, RIGHT - rightwards, LEFT - leftwards

* significant difference at $p<0.05$

Figure 3. Mean (standard deviation) of the total balance score in the unshod (grey bars) and the en pointe (black bars) condition

Spatial (sum and maximum of the CoM point displacement) and temporal (time percentage of the CoM point displacement) per quartile direction. The significance ( $p$ value) of the difference between the unshod and the en pointe conditions is noted. There was no significant difference between anterior and posterior direction (unshod relevé: $p=0.211$, 0.380, 0.947; en pointe relevé: $p=0.977,0.712,0.519$ for the sum, maximum and time percentage (\% ttotal) of the CoM point displacement, respectively). Concerning the rightwards vs. leftwards comparison, no significant difference was found for the spatial measures (unshod relevé: $p=0.997$, 0.712; en pointe relevé: $p=0.946,0.796$ for the sum and maximum of the CoM point displacement, respectively). However, the leftwards $\%$ ttotal was significantly greater compared with rightwards in the unshod relevé $(p=0.037)$, with no significant difference in the en pointe condition ( $p=0.937)$ (paired $t$-tests, separately for each variable, were applied for all bi-directional comparisons)

\section{Relative - absolute reliability}

\section{Total balance score}

The reliability indices imply a consistently lower relative and a consistently higher absolute reliability of the total balance score in the unshod compared with the en pointe condition, both in the accumulated and paired trials (Figure 4). The trial accumulation indicates a minimum of 6 trials in the unshod relevé and just 2 trials in the en pointe relevé for significant relative reliability. The paired trials reliability was not significant in the unshod condition, whereas all consecutive pairs indicated significant reliability in the en pointe condition. The absolute reliability points at an about triple SEM\% in the en pointe compared with the unshod relevé consistently for all trial accumulations except the first 2 ones. The mean \pm standard deviation of total balance score SEM\% equalled, in the unshod and the en pointe relevé, respectively, $9.5 \pm 1.2 \%$ 

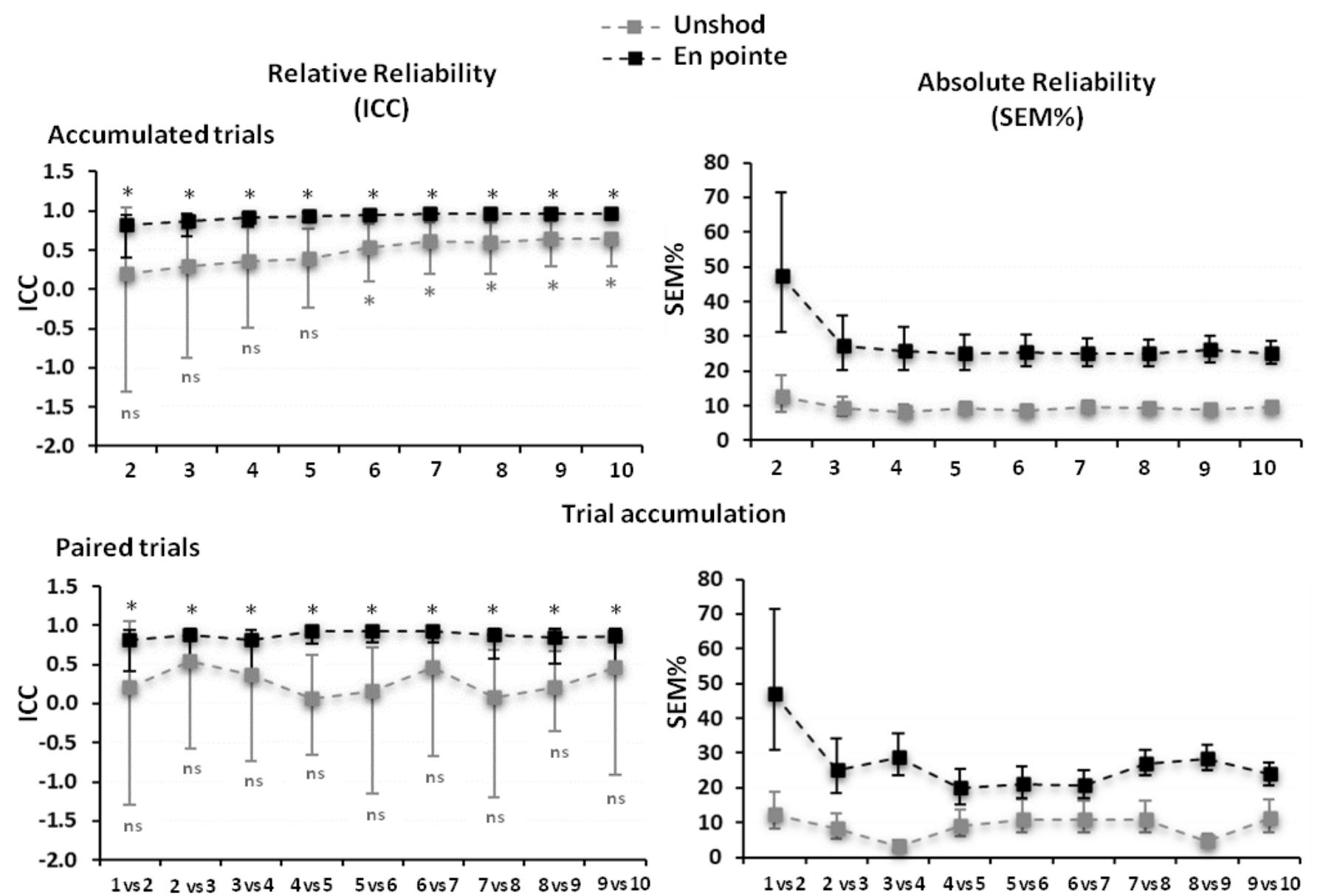

Pairs of trials

ICC - intraclass correlation coefficient, SEM\% - percentage standard error of measurement, ns - non-significant ICC * significant ICC

Figure 4. Relative (ICC) and absolute (SEM\%) total balance score for the accumulated and paired trials in the unshod (grey markers) and the en pointe (black markers) conditions. The error bars indicate the lower and upper bound of the $95 \%$ confidence intervals

and $27.9 \pm 7.2 \%$ for overall accumulated trials and 9.0. $\pm 3.2 \%$ and $26.9 \pm 8.3 \%$ for overall paired trials (Figure 4).

\section{Directional variables}

The directional variables demonstrated cases of significant fair to good reliability for the sum of the CoM displacement, and excellent reliability for the maximum of CoM displacement (in the accumulated and paired trials), whereas the time percentage of the CoM displacement presented overall non-significant reliability. However, no consistent pattern of significant reliability was demonstrated either for trial accumulation or for pairs of trials (Table 1, Figure 5). The absolute reliability of the directional variables (Figure 6) indicates a decrease and a stabilization of SEM\% after a 6-trial accumulation; however, the SEM\% of the directional variables is rather too high in both the unshod and the en pointe relevé (mean \pm standard deviation of SEM\% across all 4 directions for the sum, the maximum, and the time percentage of the CoM point displacement, respectively: overall for accumulated trials: $114.1 \pm$ $20.6 \%, 105.5 \pm 35.8 \%$, and $109.4 \pm 15.1 \%$; overall for paired trials: $106.1 \pm 25.3 \%, 96.5 \pm 35.9 \%$, and $115.7 \pm 29.2 \%)$.

\section{Discussion}

The purpose of this study was to evaluate the reliability of assessing ballet dancers' postural stability in the unshod and the en pointe relevé position with a smartphone application.

As expected, postural stability was significantly lower (by about 48\%) in the en pointe compared with the unshod relevé position. Similarly, all CoM spatial 


\section{Relative Reliability}

\section{Sum of CoM displacement}

\section{Max of CoM displacement}

\%Time of CoM displacement
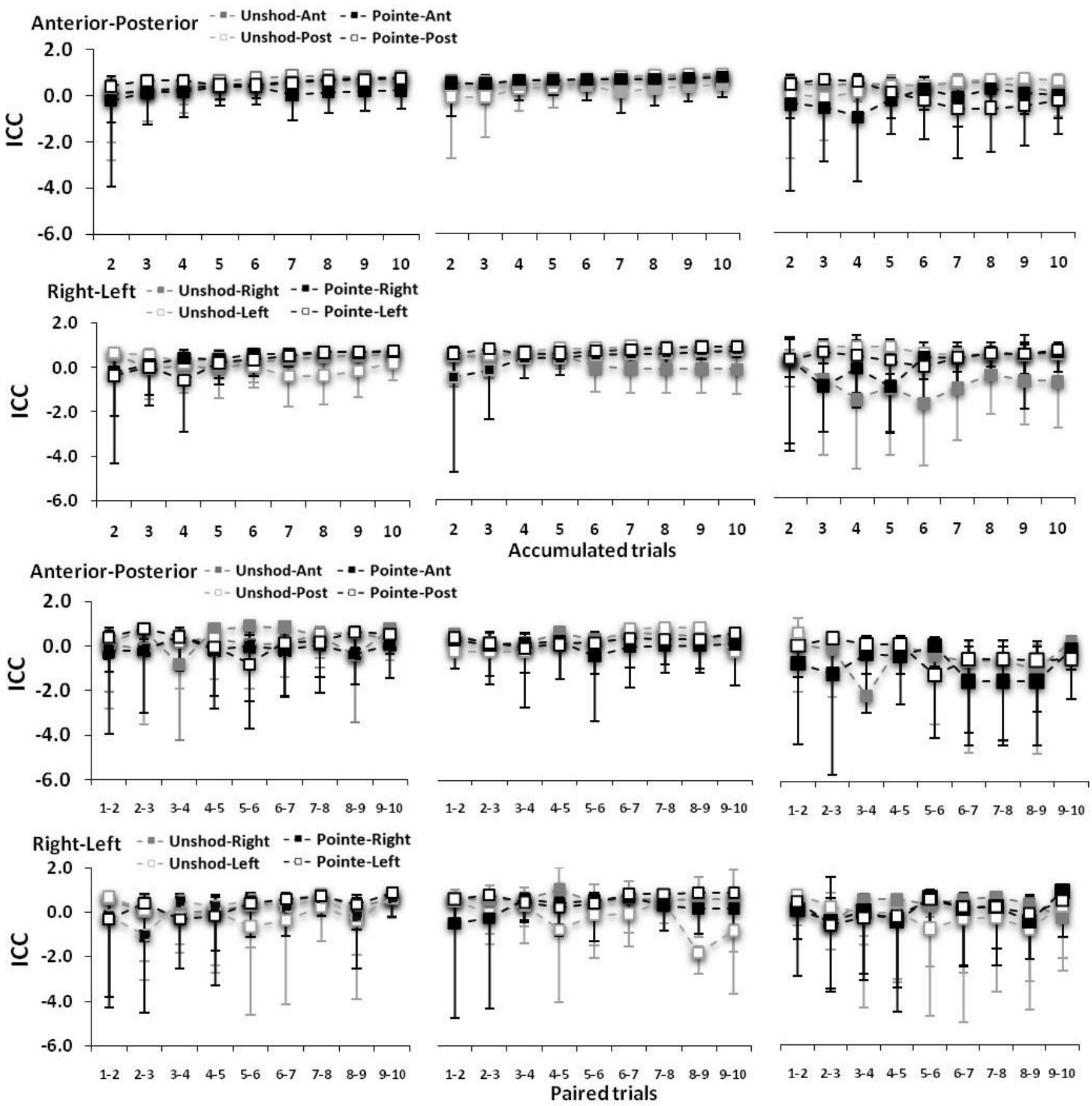

CoM - centre of mass, ICC - intraclass correlation coefficient,

Ant - anteriorly, Post - posteriorly, Right - rightwards, Left - leftwards

Figure 5. Relative reliability (ICC) of the directional variables for the accumulated and paired trials in the unshod (grey markers) and the en pointe (black markers) relevé conditions. The error bars indicate the lower and upper bound of the $95 \%$ confidence intervals. The significance of ICCs is presented in Table 1 
F. Paderi, A. Emmanouil, E. Rousanoglou, Smartphone reliability for ballet stability estimation

Table 1. Relative reliability significance $(p<0.05)$ for the directional variables presented in Figure 5

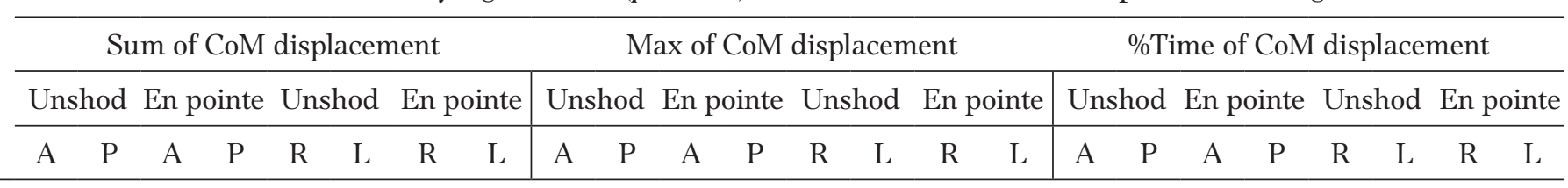

Accumulated trials

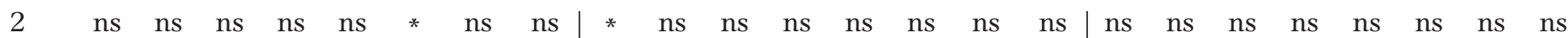

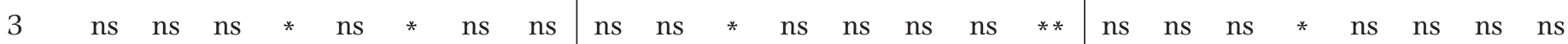

4 ns ns ns * $\quad$ ns ns ns ns $\quad * \quad n s \quad n s \quad * \quad * \quad * \quad$ ns $\quad * \quad$ ns ns ns $\quad * \quad$ ns $\quad * \quad$ ns ns

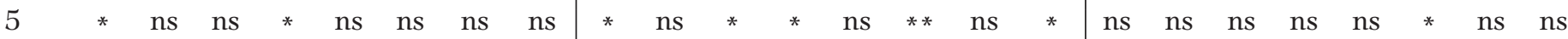

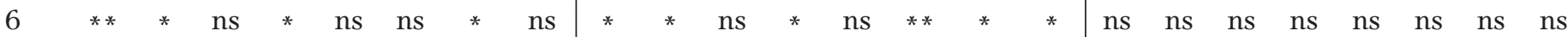

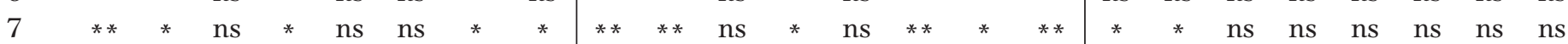

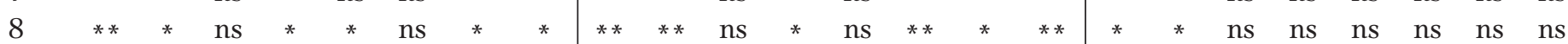

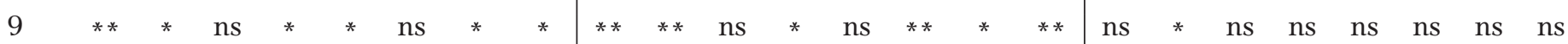

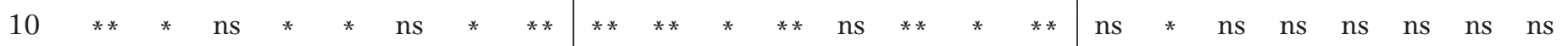

Paired trials

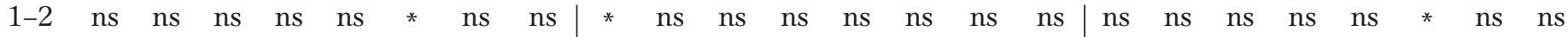

$2-3 \quad *$ ns ns * * ns ns ns ns ns ns ns ns ns ns ns * $\quad$ ns ns ns ns * $\quad$ ns ns ns ns

3-4 ns ns ns ns ns ns ns ns ns ns ns ns ns ns ns ns ns ns ns ns ns ns ns ns

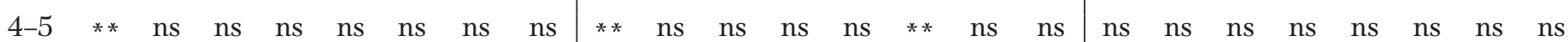

5-6 * * ns ns ns ns ns ns ns ns ns ns ns ns ns $\quad$ ns ns ns ns ns ns ns ns ns ns

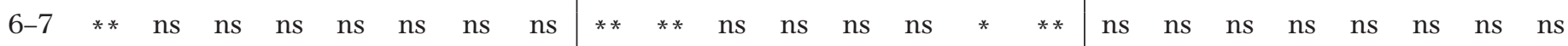

7-8 ns ns ns ns * * ns * * * * * * ns ns ns ns ns $\quad * \quad$ ns ns ns ns ns ns ns ns

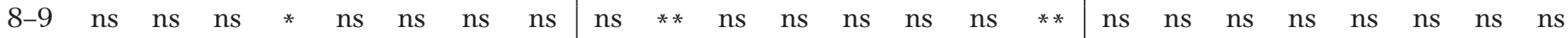

9-10 * * ns ns ns ns * ns * * ns ns ns $\quad * \quad$ ns $\quad$ ns ns $\quad * * \quad * \quad$ ns ns ns ns ns * * ns

CoM - centre of mass, A - anteriorly, P - posteriorly, $\mathrm{R}$ - rightwards, L - leftwards, ns - non-significant reliability

* fair to good reliability $(0.40>$ ICC $>0.75), * *$ excellent reliability $($ ICC $>0.75)$ (intraclass correlation coefficient [ICC]

classification in accordance with Fleiss [26])

measures indicated significantly lower postural stability in the en pointe compared with the unshod condition, whereas the directional temporal distribution of the CoM point displacement did not differ between conditions. Overall, taken an adequate number of trials, the results encourage the use of a smartphone application for testing standing postural stability in ballet dancers. This approach employs a low-cost and of high-portability tool for field assessment compared with expensive and of limited-portability laboratory equipment such as the force plate gold standard $[2,7$, $26]$, accelerometers [3, 7, 10-12], or a motion capture system $[6,8,9]$.

The results indicate the total balance score as the most reliable postural stability measure provided by the YMED Balance Test application used in the present study, whereas the directional CoM information (spatial or temporal) appears of relative but not of absolute reliability value. The superior reliability of the total balance score over the directional CoM measures is supported by both the relative and absolute reliability indices and agrees with studies of varying smartphone reliability depending on the variable used to examine postural stability [16]. Overall, the total balance score reliability appears similar to other smartphone applications; Amick et al. [14] report excellent relative reliability with ICCs of 0.78 (SEM: 5.82) in standing tasks (full contact with the floor, double or single leg standing, 2 trials) using the Sway balance mobile application. Good to excellent relative reliability ICC indices are also reported for postural stability assessments with accelerometers (ICC: 0.62-0.71 [10], ICC: 0.736-0.972 [12]).

The reliability criteria themselves warrant careful computation and interpretation owing to the variety of computational models and the wide range of classification boundaries [25, 26]. Furthermore, one should realize the different interpretations of relative (the degree to which individuals maintain their position in a sample over repeated measurements) vs. absolute (the degree to which repeated measurements vary for individuals) reliability indices [26]. Specifically, the ICC indices in our study indicate good relative reliability for the total balance score in the unshod condition and excellent relative reliability in the en pointe condition. Nevertheless, as may be inferred from its 


\section{Absolute Reliability}

Sum of CoM displacement
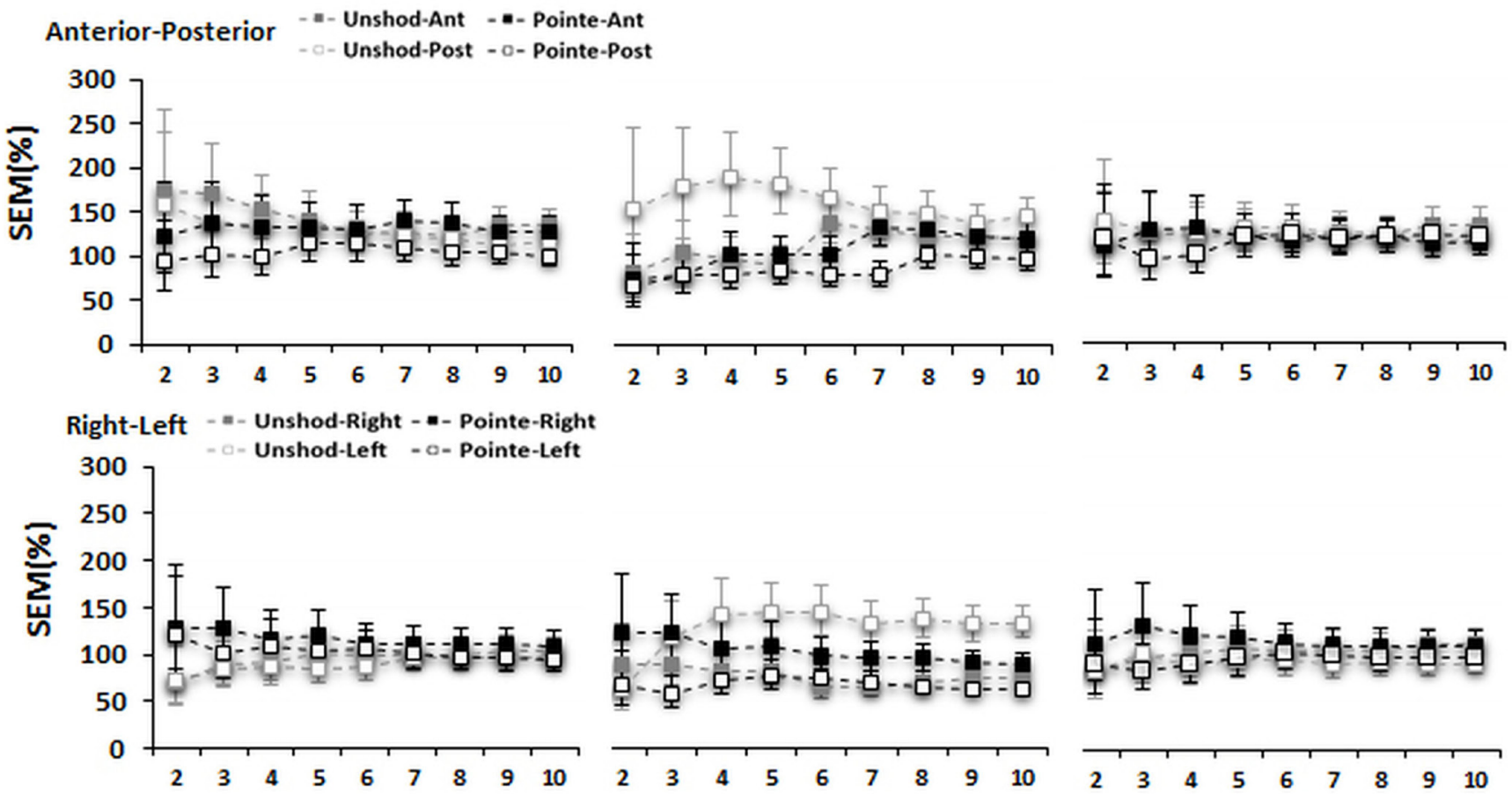

Accumulated trials
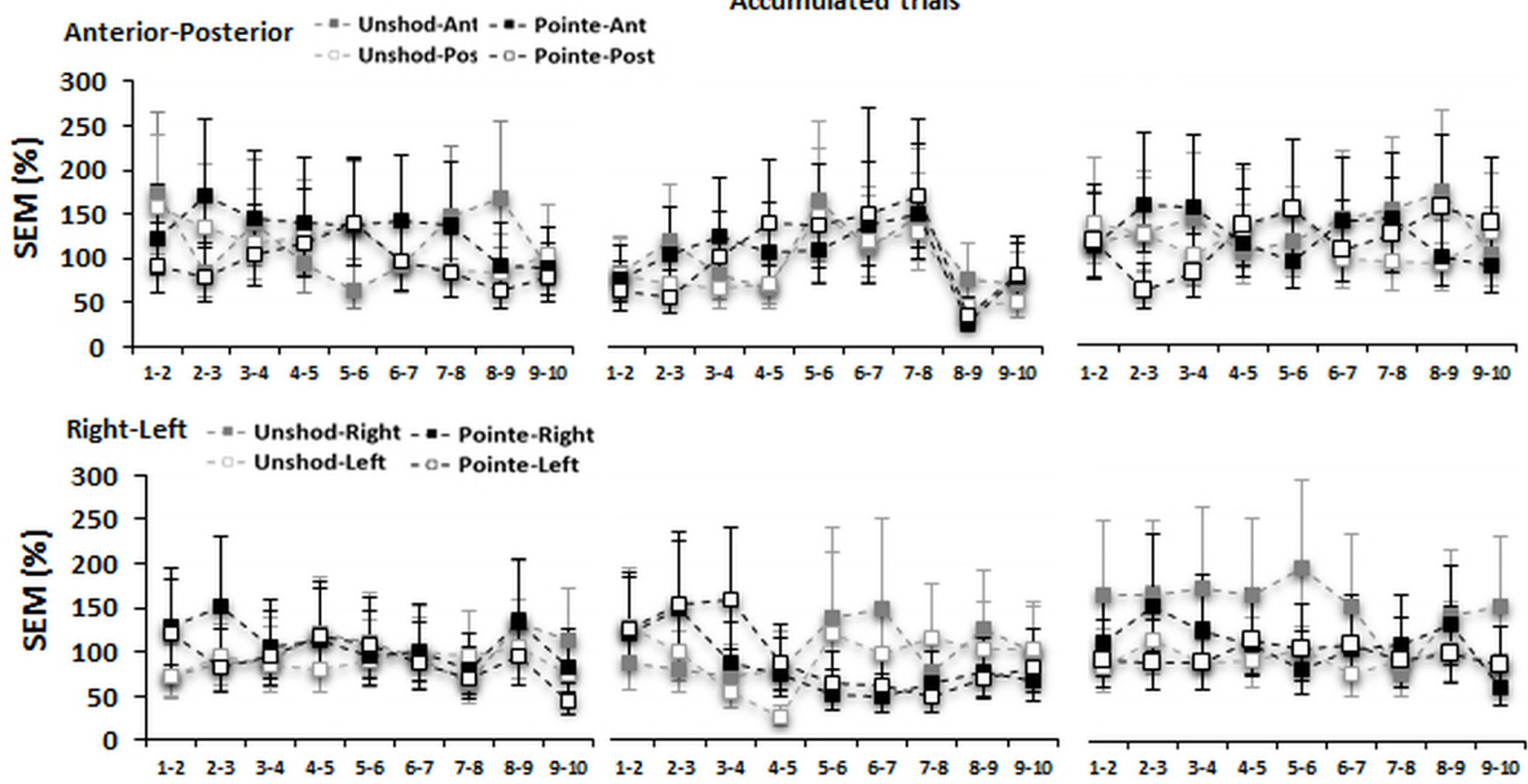

Paired trials

CoM - centre of mass, SEM\% - percentage standard error of measurement,

Ant - anteriorly, Post - posteriorly, Right - rightwards, Left - leftwards

Figure 6. Absolute reliability (SEM\%) of the directional variables for the accumulated and paired trials in the unshod (grey markers) and the en pointe (black markers) relevé conditions. The error bars indicate the lower and upper bound of the $95 \%$ confidence intervals 
F. Paderi, A. Emmanouil, E. Rousanoglou, Smartphone reliability for ballet stability estimation

about $1 / 3$ lower SEM $\%$, the absolute reliability of the total balance score is better in the unshod than the en pointe condition. Also, the degree of reliability of the spatial measures is not consistent in the unshod vs. the en pointe condition, indicating a directional intercondition differentiation for the sum, but not for the maximum of the CoM point displacement. Precisely, in the unshod condition, the relative reliability was fair to good in the anterior and posterior directions but poor in the medial and lateral ones, while the opposite was found for the en pointe condition. Concerning the maximum of the CoM point displacement, in both the unshod and the en pointe conditions, the relative reliability appears consistently higher (good to excellent or fair to good) in the medial and lateral directions compared with the anterior and posterior ones. Nevertheless, despite the existence of good or excellent relative reliability for the spatial directional variables, their high SEM\% indicates that the directional absolute reliability of the YMED Balance Test is poor.

One may argue that a methodological bias may underlie the directional reliability differences, as well as the greater leftward rather than rightward spatial and temporal measures. Bias in research is an important issue; as it may not be totally eliminated, it is important to understand it in order to consider strategies to minimize it. However, an accelerometer sensor bias or an environmental attractor (i.e. the examiner's position or a wall nearby) are not likely to explain the directional differences observed in the present study. Before the initiation of the measurements, the accelerometer sensor of the smartphone was calibrated and the procedures described by Ma et al. [20] were applied for static bias testing. Furthermore, the examiner was standing behind and not laterally to the participant to monitor the smartphone's screen, and although a wall existed nearby, it was not consistently on the right or left side of the participants. The stance leg dominance could possibly associate with the directional difference (the left leg was reported as the preferred stance leg for work en pointe and also the left leg was indicated as the stance dominant one in the leg dominance test). Indeed, leg dominance appears to differentiate the asymmetrical function of the feet (by how one foot performs the mobilizing function, emphasizing the precision of the movement, whereas the other foot stabilizes the upper body, providing support for the tracing foot) [29]. However, leg dominance does not appear to influence postural stability during the quiet upright stance or the single-legged stance [29].

Force plates are considered the gold standard for postural stability evaluation and allow a high test-retest reliability, but they are generally expensive and inaccessible for field testing [11, 15, 16]. In turn, accelerometers demonstrate reliability indices that are comparable with those allowed by force plates [11]. Indeed, it is not of surprise that some researchers suggest accelerometers as superior to force plates owing to their ability to quantify human movement in natural environments [11]. Thus, because of their built-in accelerometers, smartphone applications may provide a valid alternative to the force plate gold standard for a field setting evaluation of postural stability $[9,11,14,16,17]$. Nevertheless, the reliability of measurements acquired with smartphone applications remains an issue of concern [9, 14, 16, 17].

The mobile phone was positioned at the lumbar level (CoM approximation) in accordance with the YMED Balance Test guidelines, coinciding with the majority of research using accelerometers to assess postural stability [11, 22]. It must be noted, though, that other body placements are also applied in smartphone application studies (i.e. thorax, knee, ankle), with the degree of reliability varying not only owing to placement but also in conjunction with the type of standing test $[9,13,18]$. For instance, depending on the type of balance test (static or dynamic), reliability might be higher in the thoracic than the lumbar level [9]. Similarly, depending on the degree of test difficulty, the ankle location may provide a greater detection sensitivity, easiness of application, and feasibility, but this would be at the cost of potentially greater asymmetry between the left and right legs [18]. Shah et al. [18] attribute the lower reliability of the trunk placement to the low sampling rate of their smartphone built-in accelerometer (i.e. $14-15 \mathrm{~Hz}$ ). They associate the low sampling rate with a potential failure of trunk movement detection if the individual's balance is not significantly challenged. Thus, the sampling rate should be taken into consideration when comparing results with other studies, particularly when research-grade accelerometers are used, which allow a sampling rate of up to 1000 samples per second [22]. However, as mobile phone technology advances and the embedded accelerometers improve, they may be able to provide higher sampling rates and more robust measures of standing balance. Indeed, the main limitation of the present study is that the measurements of the smartphone application were not compared with a gold standard such as a force plate or a research-grade accelerometer tested for validity and reliability.

The number of trials necessary to obtain a good reliability index is a concern in many studies [14, 21], even for the most reliable among assessment tools, i.e. the 
force plate gold standard, where just 2 or 3 trials may ensure excellent relative reliability [21]. Just 2 trials are also reported to provide excellent intersession relative reliability when using a smartphone application to evaluate postural stability in the typical bipedal stance [14]. However, the relevé position, and particularly the en pointe one, is not among the stances commonly examined in previous studies concerning ballet dancers' postural stability [1-5]. Thus, a total of 10 trials were selected to ensure that the number of trials would be adequate to extract safe reliability conclusions for both the unshod and the en pointe relevé stances.

As emphasized by Atkinson and Nevill [30], the higher the SEM\%, the lower the absolute reliability and the lower the precision of the obtained results. However, to the best of our knowledge, there do not appear specific criteria concerning SEM\% classification. SEM\% of $9-14 \%$ was classified by Jaworski et al. [10] as very good reliability, while values of $40 \%$ were interpreted as significantly worse (single-legged standing, inertial sensor accelerometer). Similarly, Pooranawatthanakul and Siriphorn [9] report SEM\% at about 16\% for common standing balance tests performed with a mobile phone application. Taken into account these 2 studies, we may infer very good absolute reliability of the total balance score in the unshod condition and moderate absolute reliability in the en pointe condition, with the latter most likely within a normal range owing to its extreme anatomical and mechanical configuration. Nevertheless, future studies are necessary to provide normative data concerning the reliability of assessing postural stability during the en pointe relevé position, which is not among the standing positions commonly examined in the existing literature.

\section{Conclusions}

Postural stability was significantly lower (by about $48 \%$ ) in the en pointe compared with the unshod relevé position. The total balance score is highlighted of good and excellent relative reliability in the unshod relevé, as well as of good and moderate absolute reliability in the en pointe relevé. The directional parameters of the CoM point displacement demonstrate an overall good relative reliability but their absolute reliability is rather poor. Overall, a minimum of 8 trials are suggested for reliable results in both the unshod and the en pointe relevé. The results encourage the use of a smartphone application for testing standing postural stability in ballet dancers as it constitutes a lowcost and high-portability tool for field assessment.

\section{Acknowledgements}

We thank George Vagenas, PhD, McGill University, Emeritus Professor of Statistics in PESS, School of Physical Education and Sport Science, National and Kapodistrian University of Athens, for his valuable contribution in the statistics of the manuscript.

\section{Funding}

No funding was provided for this study.

\section{Disclosure statement}

No author has any financial interest or received any financial benefit from this research.

\section{Conflict of interest}

The authors state no conflict of interest.

\section{Author contributions}

All authors participated in the research concept and design, the collection and/or assembly of data, the data analysis and interpretation, as well as in writing, critical revision, and final approval of the article.

\section{References}

1. Da Silveira Costa MS, de Sá Ferreira A, Felicio LR. Static and dynamic balance in ballet dancers: a literature review. Fisioter Pesq. 2013;20(3):299-305; doi: 10.1590/S1809-29502013000300016.

2. De Mello MC, de Sá Ferreira A, Felicio LR. Postural control during different unipodal positions in professional ballet dancers. J Dance Med Sci. 2017;21(4):151155; doi: 10.12678/1089-313X.21.4.151.

3. Hinton-Lewis CW, McDonough E, Moyle GM, Thiel DV. An assessment of postural sway in ballet dancers during first position, relevé and sauté with accelerometers. Procedia Eng. 2016;147:127-132; doi: 10.1016/j. proeng.2016.06.201.

4. Janura M, Procházková M, Svoboda Z, Bizovská L, Jandová S, Konečný P. Standing balance of professional ballet dancers and non-dancers under different conditions. PLoS One. 2019;14(10):e0224145; doi: 10.1371/ journal.pone.0224145.

5. Lin C-F, Lee I-J, Liao J-H, Wu H-W, Su F-C. Comparison of postural stability between injured and uninjured ballet dancers. Am J Sports Med. 2011;39(6): 1324-1331; doi: 10.1177/0363546510393943.

6. Lin C-F, Su F-C, Wu H-W. Ankle biomechanics of ballet dancers in relevé en pointé dance. Res Sports Med. 2005;13(1):23-35; doi: 10.1080/15438620590922068.

7. Lee C-H, Sun T-L. Evaluation of postural stability based on a force plate and inertial sensor during static balance measurements. J Physiol Anthropol. 2018;37(1): 27; doi: 10.1186/s40101-018-0187-5.

8. Newton RU, Neal RJ. Three-dimensional quantification of human standing posture. Gait Posture. 1994;2(4): 205-212; doi: 10.1016/0966-6362(94)90105-8. 
F. Paderi, A. Emmanouil, E. Rousanoglou, Smartphone reliability for ballet stability estimation

9. Pooranawatthanakul K, Siriphorn A. Comparisons of the validity and reliability of two smartphone placements for balance assessment using an accelerometerbased application. Eur J Physiother. 2020;22(4):236242; doi: 10.1080/21679169.2019.1598489.

10. Jaworski J, Ambroży T, Lech G, Spieszny M, Bujas P, Żak M, et al. Absolute and relative reliability of several measures of static postural stability calculated using a GYKO inertial sensor system. Acta Bioeng Biomech. 2020;22(2):94-99; doi: 10.37190/ABB-01502-2019-02.

11. Moe-Nilssen R, Helbostad JL. Trunk accelerometry as a measure of balance control during quiet standing. Gait Posture. 2002;16(1):60-68; doi: 10.1016/s09666362(01)00200-4.

12. Saunders NW, Koutakis P, Kloos AD, Kegelmeyer DA, Dicke JD, Devor ST. Reliability and validity of a wireless accelerometer for the assessment of postural sway. $\mathrm{J}$ Appl Biomech. 2015;31(3):159-163; doi: 10.1123/jab. 2014-0232.

13. Steinberg N, Adams R, Waddington G, Karin J, Tirosh O. Is there a correlation between static and dynamic postural balance among young male and female dancers? J Mot Behav. 2017;49(2):163-171; doi: 10.1080/0022 2895.2016.1161595.

14. Amick RZ, Chaparro A, Patterson JA, Jorgensen MJ. Test-retest reliability of the Sway Balance Mobile Application. J Mob Technol Med. 2015;4(2):40-47; doi: 10.7309/jmtm.4.2.6.

15. Park S-D, Kim J-S, Kim S-Y. Reliability and validity of the postural balance application program using the movement accelerometer principles in healthy young adults. Phys Ther Kor. 2013;20(2):52-59; doi: 10.12674/ ptk.2013.20.2.052.

16. Polechoński J, Nawrocka A, Wodarski P, Tomik R. Applicability of smartphone for dynamic postural stability evaluation. BioMed Res Int. 2019;2019:9753898; doi: 10.1155/2019/9753898.

17. Moral-Munoz JA, Esteban-Moreno B, Herrera-Viedma E, Cobo MJ, Pérez IJ. Smartphone applications to perform body balance assessment: a standardized review. J Med Syst. 2018;42(7):119; doi: 10.1007/s10916018-0970-1.

18. Shah N, Aleong R, So I. Novel use of a smartphone to measure standing balance. JMIR Rehabil Assist Technol. 2016;3(1):e4; doi: 10.2196/rehab.4511.

19. Bickle C, Deighan M, Theis N. The effect of pointe shoe deterioration on foot and ankle kinematics and kinetics in professional ballet dancers. Hum Mov Sci. 2018;60:72-77; doi: 10.1016/j.humov.2018.05.011.

20. Ma Z, Qiao Y, Lee B, Fallon E. Experimental evaluation of mobile phone sensors. $24^{\text {th }}$ IET Irish Signals and Systems Conference (ISSC 2013), Letterkenny, 20-21 June 2013; doi: 10.1049/ic.2013.0047.

21. Pinsault N, Vuillerme N. Test-retest reliability of centre of foot pressure measures to assess postural control during unperturbed stance. Med Eng Phys. 2009;31(2): 276-286; doi: 10.1016/j.medengphy.2008.08.003.
22. Neville C, Ludlow C, Rieger B. Measuring postural stability with an inertial sensor: validity and sensitivity. Med Devices. 2015;8:447-455; doi: 10.2147/MDER. S91719.

23. Seimetz C, Tan D, Katayama R, Lockhart T. A comparison between methods of measuring postural stability: force plates versus accelerometers. Biomed Sci Instrum. 2012;48:386-392.

24. Himes JH. Reliability of anthropometric methods and replicate measurements. Am J Phys Anthropol. 1989; 79(1):77-80; doi: 10.1002/ajpa.1330790108.

25. McGraw KO, Wong SP. Forming inferences about some intraclass correlation coefficients. Psychol Methods. 1996;1(1):30-46; doi: 10.1037/1082-989X.1.1.30.

26. Fleiss JL. Reliability of measurement. In: Fleiss JL, The design and analysis of clinical experiments. New York: Wiley; 1986; 1-32.

27. Weir JP. Quantifying test-retest reliability using the intraclass correlation coefficient and the SEM. J Strength Cond Res. 2005;19(1):231-240; doi: 10.1519/15184.1.

28. Hopkins WG. Measures of reliability in sports medicine and science. Sports Med. 2000;30(1):1-15; doi: 10.2165/00007256-200030010-00001.

29. Wang Z, Newell KM. Footedness exploited as a function of postural task asymmetry. Laterality. 2013;18(3): 303-318; doi: 10.1080/1357650X.2012.672423.

30. Atkinson G, Nevill AM. Statistical methods for assessing measurement error (reliability) in variables relevant to sports medicine. Sports Med. 1998;26(4):217238; doi: 10.2165/00007256-199826040-00002. 